\title{
Soluble Guanylate Cyclase Is Required during Development for Visual System Function in Drosophila
}

\author{
Sarah M. Gibbs, ${ }^{1}$ Ann Becker, ${ }^{2}$ Robert W. Hardy, ${ }^{2}$ and James W. Truman ${ }^{1}$ \\ ${ }^{1}$ Graduate Program in Neurobiology and Behavior, Department of Zoology, University of Washington, Seattle, Washington \\ 98195, and 2Departments of Biology and Neuroscience, University of California at San Diego, La Jolla, California 92093
}

\begin{abstract}
A requirement for nitric oxide (NO) in visual system development has been demonstrated in many model systems, but the role of potential downstream effector molecules has not been established. Developing Drosophila photoreceptors express an NO-sensitive soluble guanylate cyclase (sGC), whereas the optic lobe targets express NO synthase. Both of these molecules are expressed after photoreceptor outgrowth to the optic lobe, when retinal growth cones are actively selecting their postsynaptic partners. We have previously shown that inhibition of the NO-cGMP pathway in vitro leads to overgrowth of retinal axons. Here we examined flies mutant for the $\alpha$ subunit gene of the Drosophila sGC (Gc $\alpha 1)$. This mutation severely reduced but did not abolish $\mathrm{GC} \alpha 1$ protein levels and NOstimulated SGC activity in the developing photoreceptors. Although few mutant individuals possessed a disorganized retinal
\end{abstract}

The formation of appropriate synaptic connections during development provides the foundation for nervous system function and behavior in the adult. Nitric oxide (NO) has been implicated as a key regulator of synaptogenic events in a number of systems, most convincingly in the developing visual system (for review, see Cramer and Sur, 1998; Mize and Lo, 2000). Pharmacological inhibition of NO synthase (NOS) prevents elimination of inappropriate retinal projections in chick (Wu et al., 1994; Ernst et al., 1999) and the proper segregation of retinal inputs to the lateral geniculate nucleus in ferret (Cramer et al., 1996; Cramer and Sur, 1999). Endothelial NOS-neuronal NOS double-knock-out mice also display increased inappropriate retinocollicular projections (Wu et al., 2000). Although these studies support a role for NO in vertebrate visual development, they do not identify potential effector molecules through which NO could be acting in vivo. However, very recent work strongly suggests that NO regulates the sublamination of retinal inputs to the lateral geniculate nucleus in ferrets by activating soluble guanylate cyclase (sGC;

Received March 14, 2001; revised July 6, 2001; accepted July 17, 2001.

This work was supported by a grant from the National Eye Institute to C. Zuker, National Institutes of Health Predoctoral Fellowship 5T32H007138 to S.M.G., and National Institutes of Health Grant NS 13079 to J.W.T. R.W.H. is an associate of the Howard Hughes Medical Institute. The isolation and molecular characterization of the $G c \alpha 1$ alleles were performed in the laboratory of Dr. Charles Zuker (Howard Hughes Medical Institute-University of California at San Diego). We thank Dr. Zuker for generously providing mutant and transgenic flies. S.M.G. thanks Dr. J. De Vente for the cGMP antibody, Drs. H. Steller and S. L. Zipursky for the chaoptin antibody, and David Baldwin (University of Washington) for assistance with electroretinograms. A.B. and R.W.H. thank Zuker laboratory members Y. L. Sun for molecular contributions and M. Socolich for the in situ localization.

Correspondence should be addressed to Sarah M. Gibbs, Department of Neuroscience, University of Minnesota, 6-145 Jackson Hall, 321 Church Street Southeast, Minneapolis, MN 55455. E-mail: gibbs016@tc.umn.edu.

Copyright (C) 2001 Society for Neuroscience $0270-6474 / 01 / 217705-10 \$ 15.00 / 0$ projection pattern, pharmacological NOS inhibition during metamorphosis increased this disorganization in mutants to a greater degree than in the wild type. Adult mutants lacked phototactic behavior, and the off-transient component of electroretinograms was frequently absent or greatly reduced in amplitude. Normal phototaxis and off-transient amplitude were restored by heat shock-mediated Gc $\alpha 1$ expression applied during metamorphosis but not in the adult. We propose that diminished SGC activity in the visual system during development causes inappropriate or inadequate formation of firstorder retinal synapses, leading to defects in visual system function and visually mediated behavior.

Key words: phototaxis; electroretinogram; nitric oxide; cGMP; photoreceptor; invertebrate
Leamy et al., 2001). A well characterized receptor for NO (Miki et al., 1977), sGCs function as heterodimers comprising a large $\alpha$ subunit and a small $\beta$ subunit, both containing putative catalytic domains (Harteneck et al., 1990; Buechler et al., 1991). NO binding to sGC stimulates production of the second messenger cGMP (Arnold et al., 1977), which can then interact with molecular targets such as protein kinases (Farber et al., 1979; Paupardin-Tritsch et al., 1986), phosphodiesterases (Hartzell and Fischmeister, 1986), and ion channels (Johnson et al., 1986; Nawy and Jahr, 1990).

Both NO and sGC play an important role in Drosophila visual system development. During metamorphosis the photoreceptors display a period of NO-sensitive sGC activity, beginning after retinal axon outgrowth and ending before synapse formation between the photoreceptors and optic lobe targets (Gibbs and Truman, 1998). A similar type of NO sensitivity is observed in other insect systems, where many developing neurons respond to NO with increases in cGMP synthesis after arrival at their postsynaptic targets (Truman et al., 1996; Ball and Truman, 1998; Schachtner et al., 1998; Wildemann and Bicker, 1999; Zayas et al., 2000). NADPH-diaphorase staining and NOS-like immunoreactivity are present in target regions of the Drosophila optic lobe throughout metamorphosis, providing a potential source of NO to sGC-expressing photoreceptors (Gibbs and Truman, 1998; Gibbs, 2001). We have previously shown that pharmacological inhibition of NO or sGC signaling in vitro during this window of sensitivity disrupts the organization of the retinal projection pattern and leads to the overgrowth of retinal axons beyond their normal targets in the medulla (Gibbs and Truman, 1998). The retinal overgrowth observed with NOS inhibition in vitro can be rescued with the addition of a cGMP analog, suggesting that sGC 
activity in the photoreceptors is sufficient to maintain retinal axons in the appropriate target region before synaptogenesis (Gibbs and Truman, 1998).

The genetic tools available in Drosophila provide an opportunity to examine sGC function in vivo. In addition, the fruit fly is a model for understanding the physiological basis of invertebrate visual system function and visually mediated behavior (Benzer, 1967; Alawi and Pak, 1971; Heisenberg, 1972; Inoue et al., 1985; Shieh et al., 1997). Genes for the sGC $\alpha(d g c \alpha 1)$ and $\beta\left(d_{c} \beta 1\right)$ subunits have been cloned from Drosophila (Yoshikawa et al., 1993; Liu et al., 1995; Shah and Hyde, 1995), and coexpression studies demonstrated that the DGC $\alpha 1 /$ DGC $\beta 1$ heterodimer can be stimulated with NO (Shah and Hyde, 1995). In the present study we examined visual system organization and function in flies carrying a mutation in the $\alpha$ subunit gene, $G c \alpha 1$. The results suggest that diminished sGC activity during metamorphosis leads to inappropriate or inadequate connections between photoreceptors and optic lobe neurons, further supporting a requirement for sGC signaling in Drosophila visual system development.

\section{MATERIALS AND METHODS}

Fly stocks. All flies were raised at $25^{\circ} \mathrm{C}$ on standard medium. For metamorphic time points, white puparia were collected and staged from the time of collection as hours after puparium formation (APF). The Gc $\alpha 1$ mutants and transgenic flies were obtained from the laboratory of Dr. Charles Zuker (University of California at San Diego). The claret $^{l}$ and brevis $^{I}$ progenitor strains came from the Bloomington Stock Center. Canton-S and w1118 strains were also used as controls. The levels of GC $\alpha 1$ protein were indistinguishable across these control strains, as determined by Western blot of adult heads (data not shown).

Isolation of guanylate cyclase mutants. Two genetic screens were performed to isolate mutations in the guanylate cyclase gene, $G c \alpha 1$, located by in situ hybridization to 99B4-5 on the cytological map. Both used a Western blot assay to detect loss of $\mathrm{GC} \alpha 1$ protein. Mutagenesis was performed by feeding claret $^{1}$ (cal $; \mathrm{F} 3$ screen) or brevis ${ }^{1}\left(b v^{1} ; \mathrm{F} 2\right.$ screen) males $25 \mathrm{~mm}$ ethylmethane sulfonate (EMS) in $1 \%$ sucrose (Grigliatti, 1986). See Flybase for descriptions of the mutations.

The F3 screen used the temperature-sensitive dominant lethal mutation $\left[l(3) D T S 4^{I}\right)$ in the TM9 balancer chromosome. Mutagenized $c a^{I}$ males were mass-mated to TM9/Bs $b$ virgin females, and the bottles were kept at $23^{\circ} \mathrm{C}$. Single F1 males were then mated to two $T M 9 / B s b$ virgin females in a vial and kept at $29^{\circ} \mathrm{C}$ for $2 \mathrm{~d}$. Vials were then cleared of the parents and returned to $29^{\circ} \mathrm{C}$ for 2 more days before being moved to $25^{\circ} \mathrm{C}$. The F2 $\mathrm{Bsb} / \mathrm{ca}$ progeny were transferred to new vials, and the resulting F3 $\mathrm{ca}$ flies were tested by Western blot for reduced or missing protein. The alleles $G c \alpha 1^{1}$ (GC 207), Gc $\alpha 1^{2}$ (GC 738) and $G c \alpha 1^{3}$ (GC782) induced in the $c a$ marked chromosome came from 11,692 F3 lines tested. Stocks were made by selecting $\mathrm{ca}^{1}$ males and females.

For the F2 screen, mutagenized $b v^{I}$ males were mass-mated to $G c \alpha 1^{1}$, $c a^{1}$ virgin females. All crosses were done at $25^{\circ} \mathrm{C}$. Single $\mathrm{F} 1$ males were crossed to two $G c \alpha 1^{1}, c a^{1}$ virgin females, and the $\mathrm{F} 2$ wild-type flies were tested for the GC $\alpha 1$ protein. Stocks were made by selecting $b v^{1}$ flies for any lines that were missing the protein. Two alleles, $G c \alpha 1^{4}$ (GC193) and $G c \alpha 1^{5}$ (GC253), induced in a $b v^{1}$ marked chromosome, came from 8445 F2 lines tested. Additional Western blots confirmed that the three lines from the F3 screen and the two lines from the F2 screen were indeed GC mutants. $G c \alpha 1^{4}$ is no longer available. $G c \alpha 1^{1}$ was sequenced and found to be a single-base change (AG/GT to $\mathrm{AG} / \mathrm{AT}$ ) in the donor sequence at the splice junction of intron 1 . This leads to an insertion of intron sequences in the cDNA and a premature termination codon (L. Sun and C. Zuker, personal communication).

Transgenic flies. A PCR fragment representing the full-length guanylate cyclase gene, confirmed by sequencing, was cloned into the $X b a$ site of a $\mathrm{P}\{\mathrm{CaSpeR}-\mathrm{hr}\}$ heat shock transformation vector (Thummel and Pirrotta, 1992). P element-mediated germ line transformation and fly manipulations were performed using standard techniques (Karess and Rubin, 1984.).

Western blot analysis. Single adult fly heads were collected from each mutant line and sonicated in SDS-Laemmeli buffer. The samples were loaded on a $10 \%$ SDS-polyacrylamide gel and transferred onto nitrocellulose using a Bio-Rad (Hercules, CA) transblot. The membrane was blocked for $1 \mathrm{hr}$ at room temperature in PBS and $0.05 \%$ Tween 20 (PBST) containing 5\% nonfat dry milk. The blots were incubated overnight at $4^{\circ} \mathrm{C}$ in PBST containing 5\% nonfat dry milk and anti-Gc $\alpha 1$ antibody, which was made against a CT peptide. After washing in PBST (four times for $5 \mathrm{~min}$ each), the membrane was incubated for $1 \mathrm{hr}$ with peroxidase-conjugated affinity-purified goat anti-rabbit antibody (Jackson ImmunoResearch, West Grove, PA). After washing with PBST (four times for $5 \mathrm{~min}$ each), the antibody complexes were detected using chemiluminescence (SuperSignal; Pierce, Rockford, IL).

NO stimulation and cGMP immunocytochemistry. The protocol for stimulation with NO donors and cGMP immunocytochemistry has been described previously (Truman et al., 1996; Gibbs and Truman, 1998). The CNS and attached eye disks were dissected from pupae at $24 \mathrm{hr}$ APF and placed in a solution of PBS containing $1 \mathrm{~mm}$ sodium nitroprusside (SNP; Sigma, St. Louis, MO) and $1 \mathrm{mM}$ isobutylmethylxanthine (IBMX; Sigma) for $15 \mathrm{~min}$. For heat shock induction experiments, dissection and stimulation with SNP and IBMX took place $2 \mathrm{hr}$ after a 45 min heat shock at $37^{\circ} \mathrm{C}$. Tissue was then fixed overnight at $4^{\circ} \mathrm{C}$ in $4 \%$ paraformaldehyde in PBS. After rinsing in PBS plus $0.3 \%$ Triton X-100 (PBS-TX), the tissue was blocked for $30 \mathrm{~min}$ at room temperature in $5 \%$ normal donkey serum in PBS-TX. Nervous systems were incubated overnight at $4^{\circ} \mathrm{C}$ with a sheep anti-cGMP antiserum (1:10,000; a gift from J. De Vente, Maastricht University, Maastricht, The Netherlands), rinsed in several changes of PBS-TX, and incubated again overnight at $4^{\circ} \mathrm{C}$ in a Texas Red-conjugated donkey anti-sheep secondary antibody (1:500; Jackson ImmunoResearch). The tissue was rinsed and mounted on poly-L-lysine-coated coverslips and dehydrated through an ethanol series. It was then cleared in xylene and mounted in DPX mountant (Fluka, Buchs, Switzerland). Optical sections were collected on a Bio-Rad MRC 600 confocal microscope and projected in NIH Image.

CNS culture and chaoptin immunocytochemistry. The CNS culture technique was described by Gibbs and Truman (1998). Briefly, CNS-eye disk complexes were dissected from white puparia and cultured in a droplet of D22 Drosophila medium (Sigma). The medium was supplemented with $7.5 \%$ fetal calf serum and a $1 \%$ final concentration of an antibiotic-antimycotic mixture (Sigma) comprising 10,000 U/ml penicillin, $10 \mathrm{mg} / \mathrm{ml}$ streptomycin, and $25 \mathrm{mg} / \mathrm{ml}$ amphotericin B. The competitive NOS inhibitor L-nitro-arginine-methylester (L-NAME) was made as a $1 \mathrm{M}$ stock solution in tissue culture-grade water and diluted to working concentrations of $1 \mathrm{~mm}$ and 100, 10, and $1 \mu \mathrm{M}$ in D22. Control cultures received the maximum volume of sterile water. Cultures were kept at $25^{\circ} \mathrm{C}$ in a humidified incubator and aerated with $95 \%$ oxygen and $5 \%$ carbon dioxide. After $24 \mathrm{hr}$, the tissue was transferred into fresh media containing $1 \mu \mathrm{g} / \mathrm{ml}$ 20-hydroxyecdysone and cultured for a remaining 72 hr. The tissue was then fixed overnight at $4^{\circ} \mathrm{C}$ in PBS containing $4 \%$ paraformaldehye.

After a series of washes in PBS with $0.3 \%$ Triton X-100, fixed tissue was incubated overnight at $4^{\circ} \mathrm{C}$ with a mouse monoclonal antibody to chaoptin (monoclonal antibody 24B10, 1:500; a gift from Dr. S. L. Zipursky, University of California, Los Angeles, CA). A peroxidaseconjugated goat anti-mouse secondary antibody was used at 1:500 (overnight, $4^{\circ} \mathrm{C}$ ). After rinsing for at least $1 \mathrm{hr}$ with several changes of PBS-TX, antibody complexes were visualized by incubating the tissue in PBS-TX containing $0.5 \mathrm{mg} / \mathrm{ml}$ diaminobenzedine (Sigma), $2 \mathrm{mg} / \mathrm{ml} \beta$-Dglucose, $0.4 \mathrm{mg} / \mathrm{ml}$ ammonium chloride, $0.03 \%$ nickel chloride, and $0.2 \mathrm{U}$ of glucose oxidase. The tissue was mounted as described above.

After culturing, nervous systems were analyzed for retinal axon overgrowth. Each experiment was repeated at least three times, and samples were scored blind as to treatment. Nervous systems were assigned a score reflecting the amount of retinal disorganization in the medulla. Parameters were as follows: 0 , normal retinal pattern; 1 , slight disorganization in pattern and crowding of photoreceptor terminals; 2 , further disruption and gaps appearing in the pattern; 3 , growth of retinal axons beyond the medulla but not the lobula; and 4, growth of retinal axons beyond the lobula. For more details, see Gibbs and Truman (1998). Each experiment was repeated at least three times, and samples were scored blind as to treatment. A mean score was then calculated for each group, and the results were analyzed using StatWorks (Cricket Software, Malvern, PA). In addition, the percentages of total nervous systems showing retinal axon growth beyond the medulla were compiled for mutant and wildtype nervous systems. These data were pooled and used to calculate the percent change in retinal axon overgrowth in the mutants in relation to the wild type (percentage of total $G c \alpha 1^{1}$ mutant nervous systems showing retinal overgrowth/percentage of total wild-type nervous systems 
showing retinal overgrowth). The statistical significance of these differences was determined using Fisher's exact test.

Phototaxis and geotaxis assays. Behavioral assays for fast phototaxis were performed in the dark, using a countercurrent apparatus and protocol as described by Benzer (1967). Eleven $18 \times 150 \mathrm{~mm}$ plastic test tubes were joined by a sliding Lucite rack (five on top and six on bottom). The apparatus was laid horizontally in front of a fluorescent light source that was horizontal and perpendicular to the tubes. Trials were performed with mixed male-female populations of $\sim 100$ flies 1 week after eclosion. After $10 \mathrm{~min}$ of dark adaptation, flies were given $15 \mathrm{sec}$ to move from one tube into the next toward the light before being transferred horizontally to the neighboring tube. For testing negative phototaxis, the apparatus was placed in the reverse orientation, with the light source behind the flies. The distribution of flies in all six tubes was determined after five runs and calculated as a percentage of the total population per tube. Three trials were performed for each strain of flies $(\sim 300$ flies total per strain). SEs were calculated from these averages. Geotaxis assays were performed in total darkness, orienting the countercurrent apparatus vertically. Populations of $\sim 30$ flies were used per trial and given 15 sec per run. As with the phototaxis assays, three trials were run with three different populations of flies per strain. The distributions were then calculated and averaged for each strain.

Heat shock experiments were performed with flies carrying the $G c \alpha 1^{1}$ allele and a wild-type $G c \alpha 1$ transgene under heat shock control. Two sets of flies carrying the Gc $\alpha 1$ transgene and one set of claret $^{1}$ controls were raised at $18^{\circ} \mathrm{C}$. It was determined that a heat shock of $45 \mathrm{~min}$ at $37^{\circ} \mathrm{C}$ produced maximum levels of transgene expression at 3-4 hr after heat shock, as assayed by Western blot (Fig. $1 A$ ) and NO-induced cGMP immunocytochemistry, which was maintained until 6-7 hr after heat shock, when it began to decline (S. M. Gibbs, unpublished data). For the phototaxis experiments, $45 \mathrm{~min}$ heat shocks at $37^{\circ} \mathrm{C}$ were delivered every $8 \mathrm{hr}$ for the first $48 \mathrm{hr}$ of metamorphosis to one group of $h s-g c^{+} ; G c \alpha 1^{1}$ mutants. In the acute experiments, a single 45 min heat shock was delivered to adult flies, and they were tested for phototaxis $3 \mathrm{hr}$ later, when transgene expression would be peaking. In the final experiment, 45 min heat shocks were given every $12 \mathrm{hr}$ over $48 \mathrm{hr}$ to adult flies, and they were tested for phototaxis $24 \mathrm{hr}$ after the last heat shock.

Electroretinograms. Adult males 4-7 d old were anesthetized with $\mathrm{CO}_{2}$. The legs and wings were removed, and the fly was immobilized and dark-adapted for $10 \mathrm{~min}$. Extracellular recordings were performed using a glass microelectrode filled with physiological saline (in mM: $130 \mathrm{NaCl}$, $4.7 \mathrm{KCl}$, and $1.9 \mathrm{CaCl}_{2}$ ). The recording electrode was always placed subcorneally in the center of the eye; a second electrode in the thorax served as the reference electrode. The light stimulus was a $565 \mathrm{~nm}$ green light-emitting diode (LED) positioned $2 \mathrm{~cm}$ from the eye. Light pulses of $1 \mathrm{sec}$ were presented at varying intensities. Electroretinogram (ERG) responses were collected using a computer sampling at $500 \mathrm{~Hz}$ (LabView), and analysis was performed in LabView. Heat shock experiments were performed as described for the phototaxis assays. Mutant and heat-shocked flies or controls were always recorded in an alternating sequence with the same electrode.

\section{RESULTS}

\section{A mutation in Gco1 decreases sGC activity in the visual system during metamorphosis}

Genetic mutants in $G c \alpha 1$ were produced with EMS mutagenesis. Four alleles of $G c \alpha 1$ were recovered. On the basis of decreases in intensity of GC $\alpha 1$ immunoreactivity on a Western blot of adult heads, the allelic series was $G c \alpha 1^{5}=G c \alpha 1^{1}=G c \alpha 1^{3}>G c \alpha 1^{2}$. The differences in the levels of GC $\alpha 1$ protein were virtually indistinguishable among $G c \alpha 1^{5}, G c \alpha 1^{1}$, and $G c \alpha 1^{3}$ (data not shown). Almost all of the following experiments were performed with $G c \alpha 1^{1}$, the first to be isolated. In a Western blot of protein extract from wild-type adult heads, the anti-GC $\alpha 1$ antibody recognized a band of $\sim 76 \mathrm{kDa}$, approximately the size reported previously (Liu et al., 1995; Shah and Hyde, 1995). In contrast, GC $\alpha 1$ antibody staining was greatly reduced in $G c \alpha 1^{1}$ flies (Fig. $1 A$, third lane). A transgene containing the wild-type $G C \alpha 1$ was expressed with a $1 \mathrm{hr}$ heat shock in the $G c \alpha 1^{1}$ mutant, which increased the anti-GC $\alpha 1$ staining to wild-type levels (Fig. $1 A$, fourth lane, arrow). The $G c \alpha 1^{1}$ mutation not only reduced GC $\alpha 1$
A
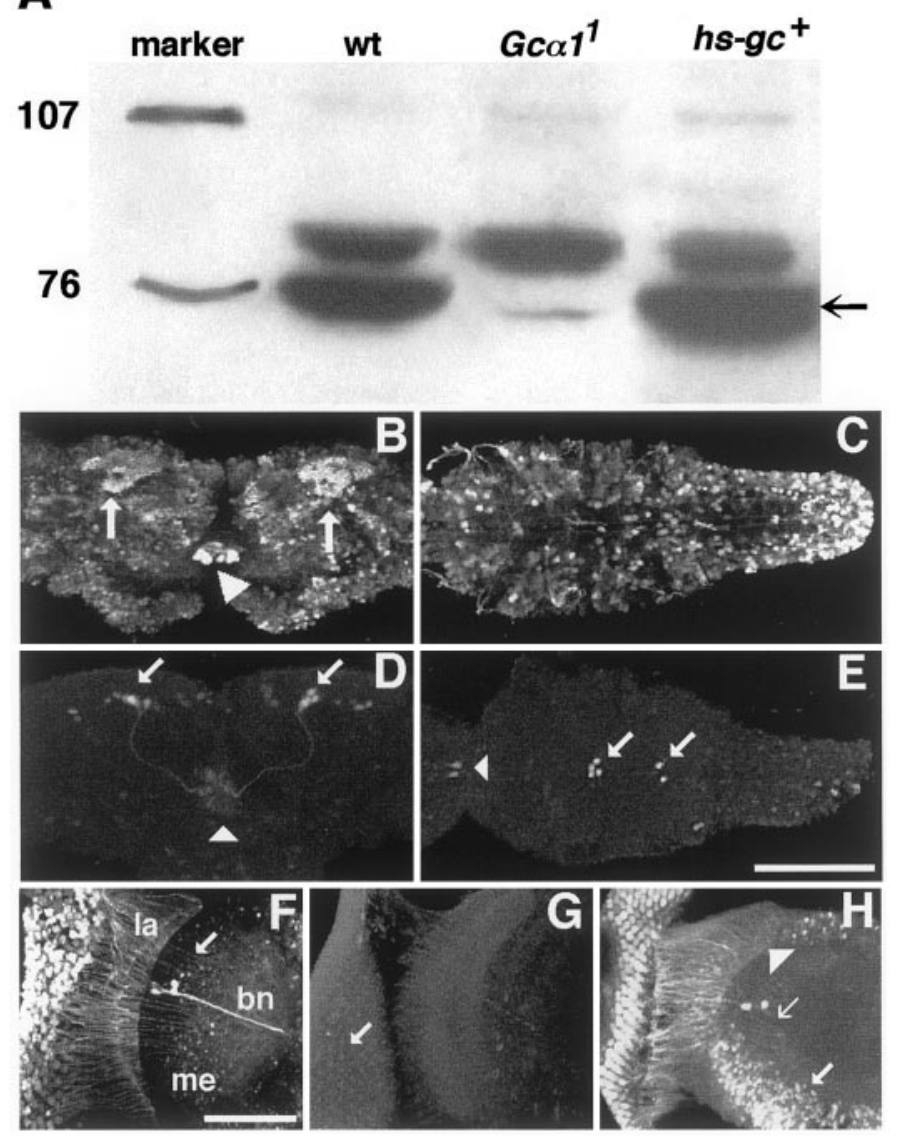

Figure 1. GC $\alpha 1$ expression and activity in wild-type and mutant flies. $A$, Western blot analysis of GC $\alpha 1$ expression. The polyclonal Gc $\alpha 1$ antibody recognized a band of $\sim 76 \mathrm{kDa}$ in adult wild-type heads (wt, second lane). Anti-Gc $\alpha 1$ staining was greatly reduced in heads from $G c \alpha 1^{1}$ mutant adults (third lane). Heat shock-induced expression of a Gc $\alpha 1$ transgene restores GC $\alpha 1$ staining in $G c \alpha 1^{1}$ (fourth lane, arrow). B-H, cGMP production in the CNS at $24 \mathrm{hr}$ APF, as revealed by cGMP antibody staining after treatment with $1 \mathrm{mM}$ SNP and IBMX. $B$, cGMP-IR was present in the wild-type brain. It is very prominent in the mushroom bodies (arrows) and in the neurons at the bast of the ring gland (arrowhead). C, cGMP-IR was also distributed throughout the wild-type ventral nervous system. $D$, cGMP-IR was dramatically reduced in the brain of $G c \alpha 1^{1}$ mutants at $24 \mathrm{hr}$ APF. Two clusters of neurons in the medial anterior brain remained cGMP-positive (arrows), sending projections to the ring gland, which also showed faint cGMP-IR (arrowhead). E, A pair of cells in the subesophageal ganglion remained cGMP-positive in $G c \alpha 1^{1}$ (arrowhead). Three pairs of cells at the ventral midline also showed cGMP-IR (arrows). $F$, In the wild-type visual system, cGMP-IR was observed in photoreceptors R1-6 projecting to the lamina (la) and R7/8 terminating in the medulla (me, arrow). Bolwig's nerve $(b n)$ also showed cGMP-IR. $G$, cGMP-IR was greatly reduced in the visual system of $G c \alpha 1^{1}$ at $24 \mathrm{hr}$ APF after exposure to $1 \mathrm{~mm}$ SNP and IBMX. Faint staining was seen in photoreceptor cell bodies of the developing eye disk (arrow). H, NO-induced cGMP-IR was restored in photoreceptors R1-6 (arrowhead) and R7/8 (small arrow) of $G c \alpha 1^{1}$ after heat shock expression of Gcal. cGMP-IR also appeared in interneurons of the medulla (large arrow). Scale bars, $50 \mu \mathrm{m}$. $B$ and $C$ represent projected stacks of optical sections (see Materials and Methods). In $F$ and $H$, the posterior- and anterior-most sections have been omitted to show the detail of projections to the lamina and medulla.

staining on a Western blot but also greatly attenuated the synthesis of cGMP in the nervous system in response to NO (Fig. $1 D, E)$. The activity of sGC in the developing Drosophila nervous system was visualized by exposing the tissue to the NO donor 
SNP (1 mM) and the phosphodiesterase inhibitor IBMX (1 mM), followed by immunocytochemistry with an anti-cGMP antibody (Truman et al., 1996; Gibbs and Truman, 1998). At 24 hr APF, the CNS of the $G c \alpha 1^{1}$ mutants showed cGMP immunoreactivity (cGMP-IR) in only a few neurons of the central brain, suboesphageal ganglion, and ventral nerve cord in response to SNP and IBMX (Fig. 1D,E). This represents a small subset of the cGMP-positive population of cells observed in the wild-type CNS at 24 hr APF after similar treatment (Fig. 1B,C). Most cells that showed cGMP-IR in $G c \alpha 1^{1}$ appear to be the same cells that remain cGMP-positive in the wild-type CNS after treatment with the sCG inhibitor $1 \mathrm{H}$-[1,2,4-oxadiazolo-[4,3,a]quinoxalin-1-one and are frequently cGMP-positive in nervous systems that have not been exposed to SNP and IBMX (Gibbs, unpublished observations). A receptor-type guanylate cyclase has been cloned from Drosophila (Liu et al., 1995), and it is possible that the cGMP-IR in these cells reflects the activity of this GC.

The photoreceptors possess NO-sensitive sGC activity during the first half of metamorphic development, after they arrive at their optic lobe targets (Gibbs and Truman, 1998). An example of cGMP staining after SNP and IBMX exposure is shown for the visual system at $24 \mathrm{hr}$ APF (Fig. $1 F$ ). cGMP immunoreactivity was observed in the cell bodies of photoreceptors in the developing retina as well as their axons projecting into the lamina and medulla regions of the optic lobe. These represent the axons of both classes of photoreceptors, R1-6 and R7/8. The axons of Bolwig's nerve were also cGMP-positive. In contrast, a very low level of cGMP-IR was observed in the visual system of $G c \alpha 1^{1}$ after SNP and IBMX treatment at $24 \mathrm{hr}$ APF, primarily in the cell bodies of the eye imaginal disc (Fig. $1 G$, arrow). This faint cGMP-IR most likely reflects the activity of remaining low levels of $\mathrm{GC} \alpha 1$ protein in the $G c \alpha 1^{1}$ mutant (Fig. $1 A$ ). Heat shockinduced expression of a wild-type $G c \alpha 1$ transgene restored the strong response of the photoreceptors to NO in $G c \alpha 1^{1}$ at $24 \mathrm{hr}$ APF. Two hours after a single 45 min heat shock, cGMP production was observed in the photoreceptor cell bodies and axons and the larval pioneers after treatment with $1 \mathrm{~mm}$ SNP and IBMX (Fig. 1H). The intensity of cGMP-IR induced with heat shock was not observed, however, without SNP and IBMX exposure (data not shown). Surprisingly, after heat shock expression of $G c \alpha 1$, strong cGMP-IR was also observed in the medulla interneurons of the optic lobe of the $G c \alpha 1^{1}$ mutants (Fig. $1 H$, arrows). This was in contrast to the wild-type visual system, in which NO-induced cGMP accumulation is very low in the medulla at $24 \mathrm{hr}$ APF, becoming more prominent by $\sim 48 \mathrm{hr}$ APF (Fig. $1 F$ ). Because both $\alpha$ and $\beta$ subunits are required for soluble guanylate cyclase activity in response to NO (Shah and Hyde, 1995), we conclude that the GC $\beta 1$ protein must already be present in the medulla interneurons at $24 \mathrm{hr}$ APF. The delayed onset of NO sensitivity in these cells may then reflect the regulated expression of GC $\alpha 1$ rather than GC $\beta 1$.

\section{Gco $1^{1}$ mutants show minor defects in the retinal projection pattern and increased sensitivity to NOS inhibition in vitro}

We have previously shown that cells of the lamina and medulla express NOS and that pharmacological inhibition of NOS or sGC in vitro causes disorganization and overgrowth of the retinal projections in the wild-type visual system (Gibbs and Truman, 1998). On the basis of these previous in vitro results, we predicted that flies mutant for $G c \alpha 1$ would display similar defects in visual system organization. We examined the retinal projection pattern
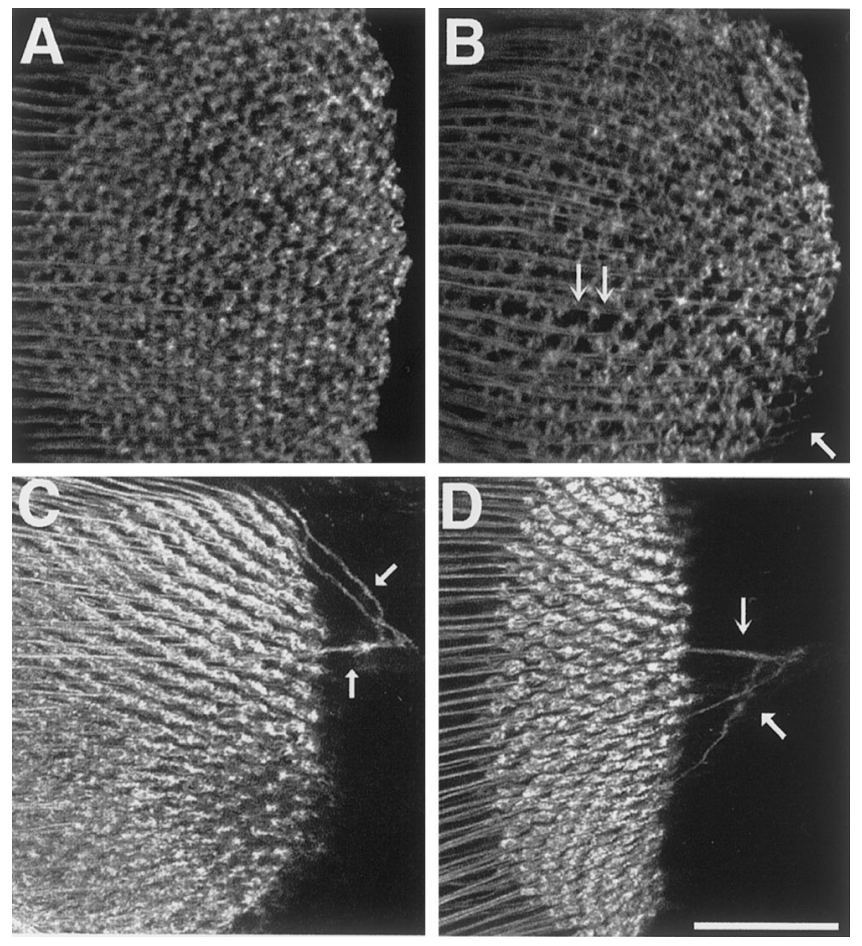

Figure 2. Gc $\alpha 1^{1}$ mutants show slight defects in visual system organization. $A$, The projections of $\mathrm{R} 7 / 8$ form a well organized pattern in the medulla of wild-type flies at $48 \mathrm{hr}$ APF, as visualized by whole-mount chaoptin immunocytochemistry. $B$, The projection pattern of $\mathrm{R} 7 / \mathrm{R} 8$ in $G c \alpha 1^{1}$ at $48 \mathrm{hr}$ APF was less well organized. Gaps in the pattern were observed (double arrows), as well as a few retinal axons projecting slightly beyond the border of the medulla (arrow). This phenotype was observed in a small percentage of mutants (6 of 130). $C, D$, Further examples of the $G c \alpha 1^{1}$ projection pattern in the medulla at $48 \mathrm{hr}$ APF, as visualized with fasciclin II immunocytochemistry. Retinal axons were seen projecting well beyond the medulla in these individuals (arrows); however, this phenotype was not typical $(n=4$ of 70$)$. Scale bar, $10 \mu \mathrm{m}$.

of R7/8 in the medulla of all four $G c \alpha 1$ alleles throughout metamorphosis and in adults, using whole-mount immunocytochemistry with antibodies to chaoptin and fasciclin II, both of which label photoreceptor axons (Zipursky et al., 1984; Schneider et al., 1995; Hiesinger et al., 1999). In general, the mutants did not show dramatic disruption of the projection pattern of $\mathrm{R} 7 / 8$, although minor defects were noted in some cases (Fig. 2; 10 of 200 flies). At $48 \mathrm{hr}$ APF, the chaoptin-stained retinal projections of a few $G c \alpha 1^{1}$ mutants were slightly disrupted when compared with the wild type (Fig. $2 A$ ), producing gaps in the pattern and extension of retinal axons slightly beyond the medullar margin and producing a "ragged" border (Fig. 2B). Similarly, staining with a fasciclin II antibody at the same stage revealed what appeared to be a few retinal axons projecting well beyond the medulla in some individuals (Fig. 2C,D). Although this phenotype was not widely observed, it was never seen in age-matched wild-type flies $(n>$ 200) and may reflect the variable penetrance of the $G c \alpha 1^{1}$ mutation.

The $G c \alpha 1^{1}$ flies express low levels of GC $\alpha 1$ protein, which is also reflected in very low levels of NO-sensitive sGC activity in the developing visual system (Fig. 1). sGC is an enzyme; thus even a small amount is capable of producing a greatly amplified cGMP signaling cascade in the presence of sufficient ligand. We therefore predicted that although this residual sGC activity was normally preventing retinal axon overgrowth in most of the 
$G c \alpha 1^{1}$ mutants, it would not be enough to compensate for decreases in NO production in vitro. CNSs and attached eye disks from wild-type (Canton-S) and mutant white puparia were placed in culture with the hormone 20-hydroxyecdysone $(1 \mu \mathrm{g} / \mathrm{ml})$, which promotes metamorphic development of the nervous system in vitro (Awad, 1995; Gibbs and Truman, 1998), or hormone plus the competitive NOS inhibitor L-NAME. After $96 \mathrm{hr}$ in culture, the tissue was then processed for chaoptin immunocytochemistry. For analysis we focused on the projection pattern of R7/8 in the medulla, which is easily resolved in whole-mount preparations. Nervous systems were assigned a score of $0-4$ based on the severity of disorganization in the projection pattern. The scores for all samples were averaged to obtain a mean score for a given treatment, called the disruption index (see Materials and Methods; Gibbs and Truman, 1998), and the percentages of nervous systems showing any retinal growth beyond the medulla were determined for mutant and wild-type nervous systems for each treatment.

A delay in neural development occurs with culturing, so that after $96 \mathrm{hr}$ in vitro, the visual system has progressed to a stage comparable to 48-50 hr APF (Gibbs and Truman, 1998). In control cultures without inhibitor, a normal, well organized projection pattern was typically seen in the medulla, and no retinal fibers were seen growing past medullar targets in either wild-type or mutant visual systems (Fig. $3 A, D$ ). Although not significantly different, the disruption index for the mutants under control conditions was slightly higher than that for the wild type, suggesting that the $G c \alpha 1^{1}$ mutation caused subtle projection pattern defects in a low percentage of individuals, as was observed with whole-mount analysis of noncultured nervous systems (Fig. 2). When a low level of L-NAME was added to the cultures $(1 \mu \mathrm{M})$, essentially no pattern disruption was observed in the medulla of wild-type nervous systems (Fig. 3B). However, this concentration of L-NAME caused the growth of many retinal axons beyond the posterior medulla of the $G c \alpha 1^{1}$ optic lobe (Fig. $3 E$ ). This effect was more severely pronounced in the mutants with $10 \mu \mathrm{M}$ L-NAME. Under these conditions, the retinal fibers produced a dense, disorganized tangle in the medulla and extended many projections into the lobula (Fig. $3 F$ ). This was not the case for wild-type nervous systems, in which treatment with $10 \mu \mathrm{M}$ L-NAME produced a slight disorganization of the projection pattern, but no retinal fibers were observed projecting beyond the medulla (Fig. 3C).

The compiled quantitative results from the in vitro experiments are shown in Figure 3, $G$ and $H$. The disruption index for $G c \alpha 1^{1}$ was significantly higher than that for wild-type controls for all tested concentrations of L-NAME (Fig. 3G; $p<0.001$, Student's unpaired $t$ test). The percent increase in $G c \alpha 1^{1}$ mutant versus wild-type nervous systems possessing retinal axons growing past the medulla for each treatment is shown in Figure $3 H$. An increase in retinal overgrowth was observed for the mutants for all treatment groups. However, significant increases in the percentage of flies with retinal axon overgrowth were seen with mutants exposed to $1 \mu \mathrm{M}$ L-NAME $(150 \%$; $p<0.02$, Fisher's exact test) and $10 \mu \mathrm{M} \mathrm{L}-\mathrm{NAME}(300 \% ; p<0.0001$, Fisher's exact test). Thus, although overall levels of disruption in the organization of the retinal projection pattern were increased in the $G c \alpha 1^{1}$ mutants (Fig. 3G), the extended growth of photoreceptors beyond the medulla was only significantly increased when nervous systems were exposed to the two lowest levels of NOS inhibitor (Fig. $3 H$ ). These results suggest that although the $G c \alpha 1^{1}$ mutants do not normally display high levels of retinal overgrowth, the decreased GC $\alpha 1$ and subsequent lower cGMP production in these flies sensitizes the developing visual system to the disruptive effects of NOS inhibition in vitro. However, the residual expression and activity of GC $\alpha 1$ in these hypomorphic mutants appear to be sufficient to normally prevent significant retinal axon overgrowth in most individuals (Figs. 1, 2).

\section{Gc 1 mutants are deficient in phototactic behavior}

To examine visual system function in the Gc 1 mutant adults, we first used a standard behavioral assay for fast phototaxis, using a countercurrent apparatus as described by Benzer (1967). In these experiments, a population of $\sim 100$ flies was placed in the first of six clear tubes, laid onto a horizontal surface, and transferred through the tubes at $30 \mathrm{sec}$ intervals as they moved toward a light source. An average of $65 \%$ of the total population of wild-type flies consistently moved toward the light after each transfer to end up in the last tube (tube 6) by the end of the trial. All four of the Gc $\alpha 1$ mutant strains showed a reduction in positive phototaxis at 1 week after eclosion when compared with the claret $^{1}$ progenitor strain (Gibbs, 2001), but the $G c \alpha 1^{1}$ mutants were the most severely compromised (Fig. 4). A combined $12-13 \%$ of $G c \alpha 1^{1}$ flies made it to the last two tubes (tubes 5 and 6) of the apparatus in repeated trials, compared with $65 \%$ of claret $^{1}$ controls. This behavior was not the result of negative phototaxis, as tested by reversing the orientation of the countercurrent apparatus relative to the light source (Fig. $4 A$ ). The profile of $G c \alpha 1^{1}$ also remained unchanged in the absence of light (Fig. $4 A$ ). Although visually mediated, phototaxis requires the integration of many behaviors (Rockwell and Seiger, 1973). However, Gc $\alpha 1^{1}$ adults showed normal performance in a geotaxis assay, demonstrating that these flies do not possess gross motor impairments (Fig. 4B).

We next investigated whether restoring sGC activity during the period of retinal innervation would improve the phototactic performance of the $G c \alpha 1^{1}$ mutants, using a heat shock-inducible Gc $\alpha 1$ transgene. In this experiment, a 45 min heat shock was given every $8 \mathrm{hr}$ for the first $48 \mathrm{hr}$ of pupal development, approximately encompassing the window of NO-sensitive cyclase activity observed in the photoreceptors. Phototaxis was tested 1 week after adult eclosion, $9 \mathrm{~d}$ after the last heat shock (Fig. $4 C$ ). At the completion of this experiment, $51 \%$ of the total population of $h s-g c^{+} ; G c \alpha 1^{1}$ adults exposed to heat shock during metamorphosis converged in the last two tubes of the countercurrent apparatus. This was compared with $13 \%$ for the non-heat-shocked $h s-g c^{+} ; G c \alpha 1^{1}$ adults and $65 \%$ for the claret $^{1}$ progenitor strain. In addition, the percentage of flies in the first two tubes was nearly identical between $\mathrm{ca}^{1}$ controls and heat-shocked $h s$ - $g c^{+} ; G c \alpha 1^{1}$ adults. Thus positive phototaxis was restored in the $G c \alpha 1^{1} \mathrm{mu}-$ tants to near wild-type levels when $G c \alpha 1$ was expressed during the first half of metamorphosis, and the phototactic profile was made to resemble that of the wild type. An improvement in positive phototaxis was not observed when adult $h s-g c^{+} ; G c \alpha 1^{1}$ flies were tested $3 \mathrm{hr}$ after a single acute heat shock (Fig. 4D). In addition, increased positive phototaxis was not seen in $h s-g_{c}^{+}$; $G c \alpha 1^{1}$ mutants that were exposed to heat shock every $12 \mathrm{hr}$ for 48 hr as adults and tested $24 \mathrm{hr}$ after the last heat shock. In this case only $17 \%$ of flies accumulated in the last two tubes, comparable with results obtained with non-heat-shocked mutants (data not shown). These results strongly support the hypothesis that the function of sGC in the phototactic response is developmental, rather than a physiological requirement for sGC activity at the time the behavior is being performed. 

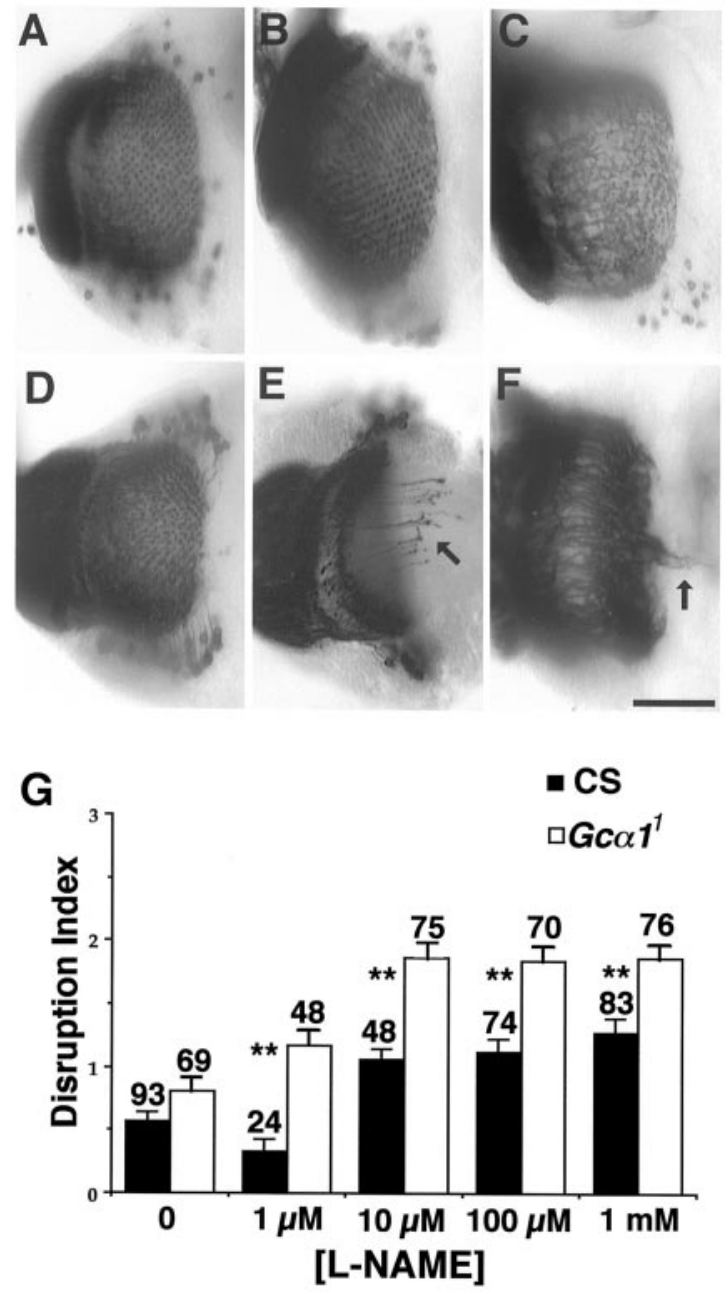

H

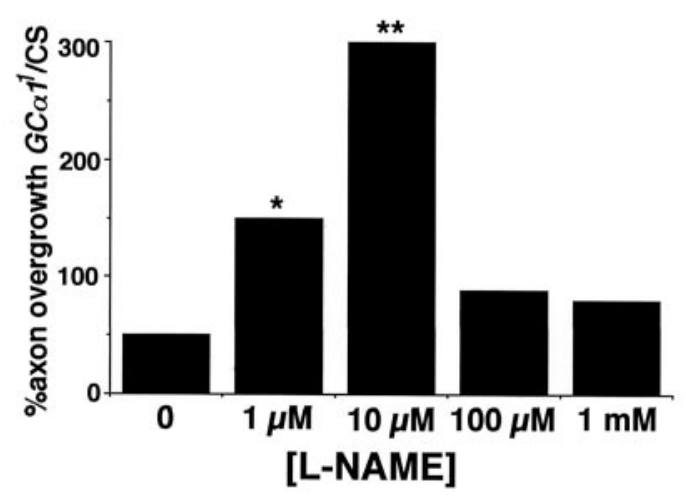

Figure 3. The visual system of $G c \alpha 1^{1}$ shows increased sensitivity to NOS inhibition in vitro. CNSs from white puparia were cultured for $96 \mathrm{hr}$ with varying concentrations of L-NAME before being processed for chaoptin immunocytochemistry. $A$, Wild-type control, cultured without L-NAME. $B$, Wild type, $1 \mu \mathrm{M}$ L-NAME. $C$, Wild type, $10 \mu \mathrm{M}$ L-NAME. $D, G c \alpha 1^{1}$ control, cultured without L-NAME. $E, G c \alpha 1^{1}$ cultured with $1 \mu \mathrm{M}$ L-NAME. The focal plane is positioned at the posterior portion of the medulla to show several retinal axons projecting beyond the posterior medulla (arrow). $F, G c \alpha 1^{1}$ cultured with $10 \mu \mathrm{M} \mathrm{L-NAME}$. The projection pattern was severely disorganized, with bundled retinal axons projecting beyond the medulla and into the lobula (arrow). Scale bar, $50 \mu \mathrm{m}$. $G$, $G c \alpha 1^{1}$ had a significantly greater disruption index than wild type for every tested concentration of L-NAME $\left({ }^{* *} p<0.001\right.$, unpaired Student's $t$ test). Numbers of optic lobes scored are given above SE bars for each group.

\section{The ERG response in Gc $\alpha 1^{1}$ mutants is reduced}

We performed ERGs in a further attempt to examine the effects of the $G c \alpha 1^{1}$ mutation on visual system function. The ERG primarily characterizes the electrophysiological response to light of a subset of photoreceptors, R1-6, and their postsynaptic cells in the lamina. The ERG consists of a corneal positive "ontransient," followed by a sustained negative wave that lasts throughout the period of illumination, and then a corneal negative "off-transient." The off-transient results from the summation of postsynaptic potentials in the monopolar neurons L1 and L2 of the lamina and can thus be used as an indicator of synaptic efficacy (Coombe, 1986).

For these experiments, two sets of $h s-g c^{+} ; G c \alpha 1^{1}$ flies were grown at $18^{\circ} \mathrm{C}$, along with a control group $\left(\right.$ claret $\left.^{l}\right)$. One set of mutants received a $45 \mathrm{~min} 37^{\circ} \mathrm{C}$ heat shock, repeated every $8 \mathrm{hr}$ from 4 to $48 \mathrm{hr}$ APF. ERGs from all three groups were recorded 5-7 d after adult eclosion. Recordings from Gc $\alpha 1^{1}$ mutants were always preceded by claret $^{1}$ controls. All the ERGs from claret $^{1}$ flies contained a normal on-transient (Fig. 5A, arrowhead), followed by a sustained depolarization of the photoreceptors in response to a $1 \mathrm{sec}$ pulse from a green LED. A large, rapid off-transient followed termination of the light stimulus in claret $^{1}$ controls (Fig. 5A, arrow). In contrast, $82 \%$ of the ERGs from the $G c \alpha 1^{1}$ flies lacked off-transients or had off-transients that were greatly reduced in amplitude (Fig. $5 B, C$, arrows). We also observed more than one peak in the reduced off-transients of some $h s-g_{c}^{+} ; G c \alpha 1^{1}$ mutants (Fig. 5D, large arrow). However, the amplitude and shape of the off-transient appeared normal in $h s-g c^{+}$; $G c \alpha 1^{1}$ flies that received heat shocks during the first half of metamorphosis (Fig. 5E, arrow). Both heat-shocked and nonheat-shocked mutants also showed a normal sustained depolarization during the light stimulus (Fig. 5B,C; data not shown), consistent with previous findings that photoreceptor function is normal in Gc $\alpha 1$ mutants (K. Scott, L. Sun, and C. Zuker, personal communication). The mean off-transient amplitude of the $G c \alpha 1^{1}$ flies $(1.54 \pm 0.25 \mathrm{mV})$ was less than half that of claret $^{1}$ controls $(3.34 \pm 0.23 \mathrm{mV})$. In contrast, $G c \alpha 1^{1}$ flies that had $G c \alpha 1$ supplied with heat shock during the first half of metamorphosis expressed a mean off-transient amplitude that was comparable with that of wild-type controls $(3.94 \pm 0.27 \mathrm{mV})$. The effect of the $G c \alpha 1^{1}$ mutation appeared to be limited to the off-transient amplitude, because both the mean duration of the off-transient peak and the mean amplitude of the sustained component were not significantly different between all three populations (data not shown). Thus, both positive phototaxis and a normal ERG trace can be restored to adult flies expressing very low levels of sGC activity when $G c \alpha 1$ is supplied developmentally as the retinal growth cones are undergoing target selection in the optic lobe.

\section{DISCUSSION}

For many years the fly has been used as a model system for understanding visual function and visually mediated behavior (Benzer, 1967; Alawi and Pak, 1971; Heisenberg, 1972; Inoue et al., 1985). More recently, the highly organized architecture of the Drosophila visual system has provided a model for understanding

$\leftarrow$

$H$, The percentage of total nervous systems containing retinal axons growing past the border of the medulla was significantly increased in the $G c \alpha 1^{1}$ mutants by $150 \%$ when cultured with $1 \mu \mathrm{M} \mathrm{L-NAME}\left({ }^{*} p<0.02\right)$ and by $300 \%$ when exposed to $10 \mu \mathrm{M} \mathrm{L-NAME}(* * p<0.0001$, Fisher's exact test). 
A
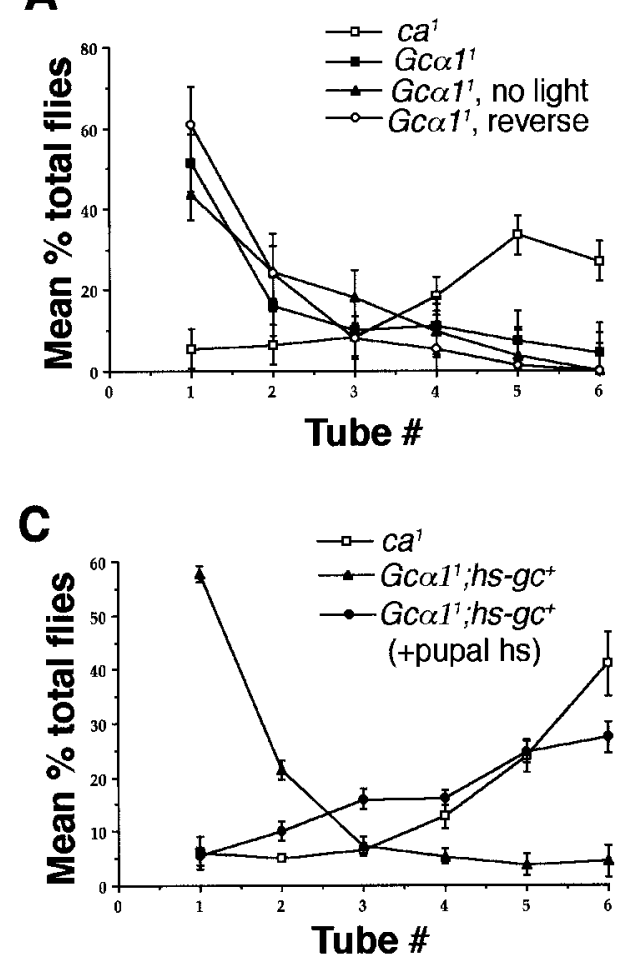

B
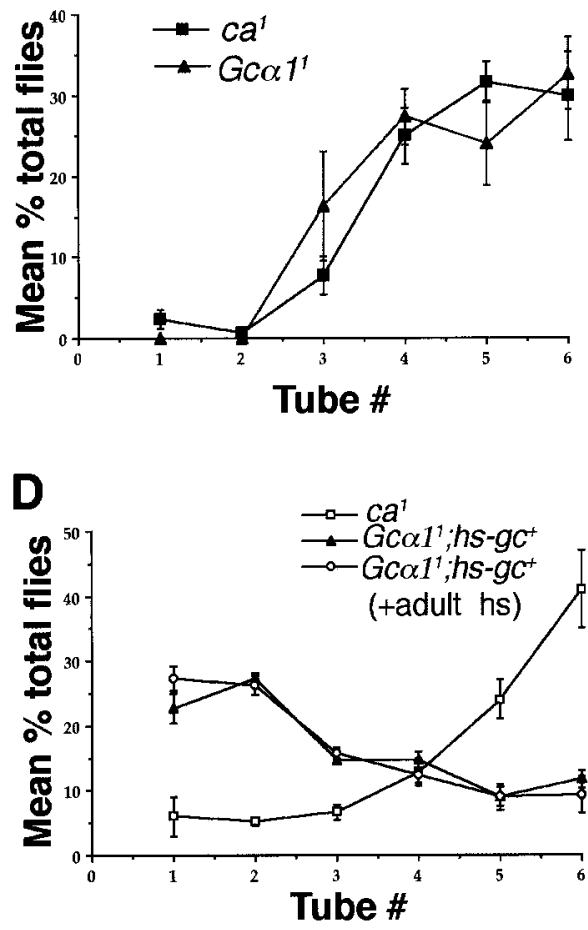

Figure 4. Heat shock expression of Gc $\alpha 1$ during metamorphosis restores positive phototaxis in $G c \alpha 1^{1}$ adults. $A$, Phototactic profiles for $G c \alpha 1^{1}$ remained weak in the absence of light (no light) and when the light source was placed in the opposite orientation to the countercurrent apparatus (reverse). $B$ $G c \alpha 1^{1}$ showed positive geotaxis that was indistinguishable from claret $^{l}$ controls. $C$, The phototactic profile of $G c \alpha 1^{1}$ more closely resembled that of the claret $^{l}$ strain after $G c \alpha 1$ was expressed with heat shock every $8 \mathrm{hr}$ during the first $48 \mathrm{hr}$ of metamorphosis. $D$, The phototactic profile of $G c \alpha 1^{1}$ did not change when $G c \alpha 1$ was expressed with a single heat shock in adult flies. Error bars indicate SE.

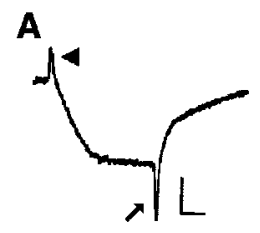

B
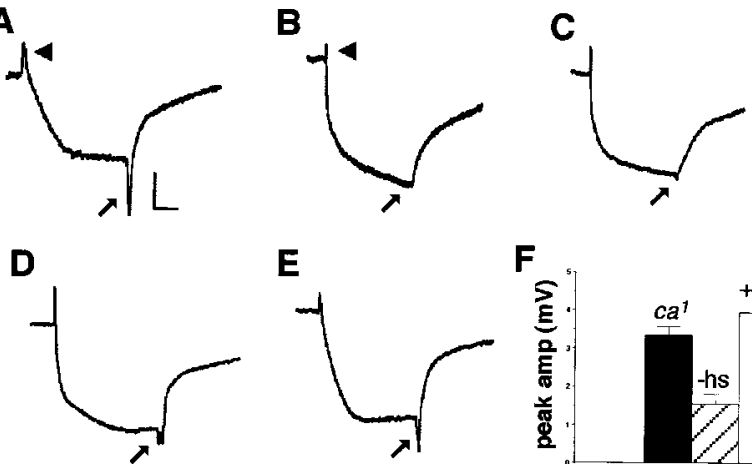

E

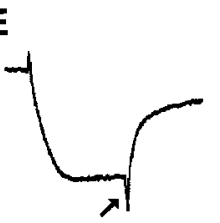

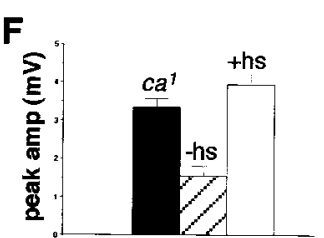

Figure 5. Gc $\alpha 1^{1}$ flies display abnormal ERGs with reduced offtransients. $A$, ERG recording from a claret $^{1}$ adult in response to a $1 \mathrm{sec}$ light pulse, showing a normal on-transient (arrowhead) and off-transient (arrow). Calibration: vertical, $3 \mathrm{mV}$; horizontal, $200 \mathrm{msec}$. B, ERG from an $h s-g_{c}^{+} ; G c \alpha 1^{1}$ mutant, showing a normal on-transient (arrowhead) and sustained depolarization. No off-transient could be detected (arrow). $C$, ERG from another $h s-g c^{+}$; $G c \alpha 1^{1}$ mutant also displaying a greatly reduced off-transient (arrow). D, ERG from a third $h s-g c^{+}$; $G c \alpha 1^{1}$ mutant, showing an off-transient of reduced amplitude containing two peaks (arrow). E, ERG from an $h s-g c^{+}$; Gc $\alpha 1^{1}$ mutant that received a heat shock every $8 \mathrm{hr}$ for approximately the first $48 \mathrm{hr}$ of metamorphosis, containing an off-transient of normal shape and amplitude (arrow). $F$, The mean amplitude of the off-transient peak in the $h s-g c^{+} ; G c \alpha 1^{1}$ mutants was significantly less $(n=17)$ than those from $h s-g c^{+} ; G c \alpha l^{1}$ mutants that received heat shocks during the first half of metamorphosis $(n=18)$ or claret $^{l}$ controls $(n=9)$. Error bars indicate SE.

axon targeting and synaptic patterning during development (for review, see Wolff et al., 1997). We have previously demonstrated the presence of NO-sensitive sGC activity in the photoreceptors between 12 and $48 \mathrm{hr}$ of metamorphosis and showed that pharmacological inhibition of $\mathrm{NO}-\mathrm{sGC}$ signaling during this temporal window disrupts the retinal projection pattern (Gibbs and Truman, 1998). In the present study, we analyzed hypomorphic

genetic mutants in the sGC $\alpha$-subunit gene $G c \alpha 1$ to further explore the role of sGC signaling in the formation of synaptic connections between photoreceptors and optic lobe interneurons. Using in vitro culture, behavioral assays, and electrophysiology, we demonstrated that decreased expression of $G c \alpha 1$ causes minor defects in visual system organization and major defects in adult visual system function. Importantly, we show that these defects could be rescued when the wild-type Gcol gene was expressed during a specific developmental period, when the location and organization of first-order retinal synapses is being established.

The $\alpha$ subunit of a soluble guanylate cyclase has been repeatedly cloned from Drosophila (Yoshikawa et al., 1993; Liu et al., 1995; Shah and Hyde, 1995; C. Zuker, A. Becker, R. W. Hardy, and L. Sun, unpublished data). However, there is controversy as to the expression pattern of Gc $\alpha 1$. Using Northern blot analysis, Yoshikawa et al. (1993) and Shah and Hyde (1995) demonstrated Gco1 mRNA in wild-type but not eyeless adult fly heads, suggesting that $G c \alpha 1$ is expressed primarily in the adult retina. Shah and Hyde (1995) also used an antibody to localize the GC $\alpha 1$ protein to the retina. Although Liu et al. (1995) showed abundant Gc 1 mRNA in a Northern blot of adult heads, the same probe failed to hybridize to adult retinal tissue in situ, and no retinal staining was found with a GC $\alpha 1$ antibody. Here we present evidence that Gcol is expressed in the photoreceptors during development. A genetic mutation that diminished $\mathrm{GC} \alpha 1$ protein levels, $G c \alpha 1^{1}$, reduced NO-induced production of cGMP in developing photoreceptors, which was restored with heat shockmediated expression of wild-type $G c \alpha 1$. Thus we conclude that $G c \alpha 1$ is normally expressed in the photoreceptors from $\sim 12$ to 48 hr APF, and we infer that the $\beta$ subunit is also present at this time. We have not determined whether the observed loss of NO sensitivity in the photoreceptors at $\sim 48 \mathrm{hr}$ APF and into adulthood (Gibbs and Truman, 1998; Gibbs, unpublished observations) is attributable to changes in Gcol expression. However, 
spatiotemporal changes in $\alpha$ and $\beta$ subunit expression may provide a molecular basis for regulating the timing of NO-sensitive sGC activity in the photoreceptors and other cells of the developing visual system.

Reports of $G c \alpha 1$ expression in the adult retina, (Yoshikawa et al., 1993; Shah and Hyde, 1995), and cGMP-mediated enhancement of the photoresponse in isolated Drosophila photoreceptors (Bacigalupo et al., 1995) implicated cGMP as a putative mediator of a phototransduction mediator in flies. Studies show that NO and cGMP can modulate the locust photoresponse (Elphick et al., 1996; Schmachtenberg and Bicker, 1999) and signaling in other insect sensory systems (Nighorn et al., 1998; Gibson and Nighorn, 2000; Ott et al., 2000). The Gc $\alpha 1$ mutants were initially generated to further establish the role of cGMP in phototransduction; however, both intracellular and extracellular recordings from these flies have revealed a normal response of the photoreceptors themselves to light (Fig. 5; K. Scott, L. Sun, and C. Zuker, personal communication). In other studies, inositol trisphosphate and diacylglycerol were shown to be primarily responsible for generating the depolarizing potential in the Drosophila retina (Zuker, 1996; Chyb et al., 1999). Our results suggest that the requirement for cGMP in the Drosophila visual system is developmental, rather than physiological. First, we never observed cGMP in the photoreceptors after $48 \mathrm{hr}$ APF, in the presence or absence of NO stimulation (Gibbs and Truman, 1998; Gibbs, unpublished data). Second, although mutant adults lacked positive phototaxis, a basic visually mediated behavior, positive phototaxis was only restored to $G c \alpha 1^{1}$ adults when $G c \alpha 1$ was supplied with heat shock during the first $48 \mathrm{hr}$ of metamorphosis, encompassing the period of observed NO-sensitive sGC activity in the photoreceptors. In contrast, phototaxis did not improve when $G c \alpha 1$ was expressed acutely or chronically in adult mutants. Our ERG results also support the hypothesis that sGC signaling is required during the first half of metamorphosis, when retinal growth cones are selecting postsynaptic partners in the optic lobe and NO-sensitive sGC activity is observed (Meinertzhagen and Hanson, 1993; Gibbs and Truman, 1998). The sustained depolarization of the photoreceptors was normal in $G c \alpha 1^{1}$ adults, but the off-transients were frequently undetectable or greatly reduced in amplitude. In addition, some off-transients contained two peaks. The postsynaptic responses of the laminar monoplar cells are responsible for generating the off-transient in Drosophila (Coombe, 1986). Thus, the decreased and aberrant off-transients observed in $G C \alpha 1^{1}$ implicates a defect in the postsynaptic response of laminar monopolar cells to retinal input in these mutants. This abnormal postsynaptic response could arise from disorganized retinal synapses, perhaps as a result of subtle deviations in growth cone behavior in the absence of normal cGMP levels. When $G c \alpha 1$ was expressed with heat shock in $G c \alpha 1^{1}$ mutants during the first half of metamorphosis, the off-transient shape and amplitude were indistinguishable from those of the wild type. The behavioral and electrophysiological results support the hypothesis that cGMP signaling is required in the photoreceptors to promote the appropriate wiring of first-order retinal synapses during metamorphosis. However, because the $G c \alpha 1^{1}$ mutation and the heat shock $G c \alpha 1$ expression were global and not eye-specific, we cannot exclude the possibility that formation of downstream connections in the optic lobe and brain also require GC $\alpha 1$. Our behavioral results in particular may reflect the effects of $G c \alpha 1$ expression on these connections. However, from 12 to 48 hr APF we observed NO-sensitive cGMP production almost exclusively in the photoreceptors and not in other visual centers until later in development (Fig. 1B; Gibbs, unpublished data). This was also the developmental window during which heat shock Gcol expression rescued both the phototactic and ERG phenotypes, which strongly suggests that the photoreceptors themselves require $\mathrm{GC} \alpha 1$ to ensure the appropriate wiring of first-order retinal synapses.

Despite the profound defects in visual system function, we did not observe a dramatic and consistent disorganization of the retinal projections in $G c \alpha 1^{1}$. This contrasts with our previous results, in which pharmacological inhibition of NO-sGC signaling caused severe disruption of the wild-type projection pattern in vitro (Gibbs and Truman, 1998). There are several possible explanations for these results. First, the $G c \alpha 1^{1}$ mutants are hypomorphs and do show low levels of GC $\alpha 1$ protein and NO-sensitive sGC activity in the photoreceptors during metamorphosis. Residual enzymatic activity of these low levels of functional sGC could produce adequate levels of retinal cGMP to prevent significant axon overgrowth provided that the ligand, NO, is present at wild-type levels. This is supported by our experiments in which nervous systems from $G c \alpha 1^{1}$ pupae were exposed to low levels of an NOS inhibitor. Under these conditions, the resulting disorganization and overgrowth of the retinal axons were much greater than in wild-type controls. These results suggest that residual GC $\alpha 1$ activity and production of cGMP by endogenous NO in $G c \alpha 1^{1}$ is sufficient to prevent overgrowth of retinal axons in vivo but cannot compensate for suppression of NO signaling in vitro. Second, we have only examined the $G c \alpha 1^{1}$ visual system at the whole-mount level. A more detailed analysis of the retinal projections and cartridge organization, perhaps using electron microscopic techniques, may reveal further architectural defects in the $G c \alpha 1^{1}$ mutants. Finally, other signaling pathways have been shown to contribute to formation of the Drosophila retinal projection pattern, and these may compensate for decreased sGC activity during visual system development. Molecules such as Hedgehog (Huang and Kunes, 1996), the EGF-like molecule Spitz (Huang et al., 1998), and N-cadherin (Lee et al., 2001) are all involved in regulating retinal axon targeting and connectivity. The most well characterized signaling pathway shown to regulate retinal targeting in Drosophila involves the recruitment of p21activated kinase (Pak) to the growth cones of growing photoreceptors by the adaptor protein Dock (Garrity et al., 1996; Rao and Zipursky 1998; Hing et al., 1999) and subsequent Pak activation by the combined signaling of Dock and the guanine nucleotide exchange factor Trio (Hing et al., 1999; Newsome et al., 2000). Interestingly, the retinal projection phenotypes of pak, dock, and trio mutants resemble the disruptive effects of NO and sGC inhibition in vitro. However, whereas the Pak-Dock pathway seems to regulate the initial stages of retinal axon outgrowth and targeting, the NO-cGMP pathway seems to be required later for maintaining the position of retinal growth cones within the target before synaptogenesis.

We propose that Gcolexpression and subsequent sGC- and NO-induced cGMP activity in the photoreceptors regulate synapse formation between photoreceptors and optic lobe neurons by exerting subtle effects on retinal growth cone behavior during cartridge assembly. Expression of NO-sensitive sGC activity was never seen during retinal axon outgrowth but only in photoreceptors that had arrived at their respective optic ganglia. This makes it unlikely that NO and cGMP are acting in a chemoattractive manner to guide retinal growth cones to the optic lobe. The metamorphic period when NOS expression in the optic lobe and NO-sensitive sGC expression in the photoreceptors was ob- 
served (12-48 hr APF; Gibbs and Truman, 1998; Gibbs, 2001) correlates temporally with when retinal growth cones are actively seeking out optic lobe cells with which they will form synaptic cartridges (Meinertzhagen and Hanson, 1993). Our current and previously published results support a model wherein NO from the target acts to stimulate cGMP synthesis in newly arrived retinal growth cones, stabilizing them and preventing further axonal extension but still allowing lateral movement within the target region. When NO production or sGC activity is inhibited pharmacologically, this stabilization is lost, and the photoreceptors resume longitudinal growth (Gibbs and Truman, 1998). Our current results show that although genetically reducing sGC activity leads to more subtle defects in visual system architecture, perhaps at the level of cartridge organization, the overall effects of this mutation on adult visual system function are profound. NO and cGMP have been proposed to regulate vertebrate visual system development by acting as effectors of activity-dependent refinement mechanisms (Ernst et al., 1999; Mize and Lo, 2000; Leamy et al., 2001). However, this is not likely to be the case in Drosophila, because the visual system can develop normally in the absence of histamine, the primary visual neurotransmitter (Melzig et al., 1998). Instead, the effects of NO-induced cGMP production may act to regulate growth cone behavior. cGMP has been shown to modulate the response of sensory growth cones and pyramidal cell dendrites to semaphorin (Song et al., 1998; Polleux et al., 2000). Additionally, sGC mediates the NO-induced behavior of filopodia in cultured Helisoma neurons (Van Wagenen and Rehder, 1999, 2000). In nematode worms, mutations in a cyclic nucleotide-gated channel affect sensory axon outgrowth during development and in adults (Coburn and Bargmann, 1996; Coburn et al., 1998). Calcium is a well documented mediator of growth cone activity (Davenport and Kater, 1992; Rehder and Kater, 1992; Gomez and Spitzer, 1999), and cGMP can produce growth cone calcium transients in vitro (Van Wagenen and Rehder, 1999; Kafitz et al., 2000). For cGMP to influence growth cone dynamics, it must ultimately affect the cytoskeleton. In cultured neuroblastoma cells, intracellular injections of cGMP cause the motile structures of growth cones to freeze and retract, whereas cAMP promotes outgrowth (Bolsover et al., 1992). More recent studies showed that cGMP-dependent protein kinase-mediated phosphorylation inactivates the small GTPase RhoA and prevents it from inhibiting myosin light chain phosphatase, thus decreasing contractility (Surks et al., 1999; Sauzeau et al., 2000; Sawada et al., 2001). Although cGMP could conceivably affect the neuronal cytoskeleton via this mechanism to modulate growth cone behavior and axon outgrowth, its role in the developing Drosophila visual system remains a question for further research.

\section{REFERENCES}

Alawi AA, Pak WL (1971) On transient of insect electroretinogram: its cellular origin. Science 172:1055-1057.

Arnold WP, Mittal CK, Katsuki S, Murad F (1977) Nitric oxide activates guanylate cyclase and increases gunaosine $3^{\prime}: 5^{\prime}$-cyclic monophosphate levels in various tissue preparations. Proc Natl Acad Sci USA 74:3202-3207.

Awad TA (1995) Endocrine regulation of neurogenesis and gliogenesis during metamorphosis of the central nervous system in Drosophila. $\mathrm{PhD}$ disseration, University of Washington.

Bacigalupo J, Bautista DM, Brink DL, Hetzer JF, O'Day PM (1995) Cyclic-GMP enhances light-induced excitation and induces membrane currents in Drosophila retinal photoreceptors. J Neurosci 15:7196-7200.

Ball EE, Truman JW (1998) Developing grasshopper neurons show variable levels of guanylyl cyclase activity on arrival at their targets. J Comp Neurol 394:1-13.
Benzer S (1967) Behavioral mutants of Drosophila isolated by countercurrent distribution. Proc Natl Acad Sci USA 58:1112-1119.

Bolsover SR, Gilbert SH, Spector I (1992) Intracellular cyclic AMP produces effects opposite to those of cyclic GMP and calcium on the shape and motility of neuroblastoma cells. Cell Motil Cytoskeleton 22:99-116.

Buechler WA, Nakane M, Murad F (1991) Expression of soluble guanylate cyclase activity requires both enzyme subunits. Biochem Biophys Res Commun 174:351-357.

Chyb S, Raghu P, Hardie RC (1999) Polyunsaturated fatty acids activate the Drosophila light-sensitive channels TRP and TRPL. Nature 397:255-259.

Coombe PE (1986) The large monopolar cells L1 and L2 are responsible for ERG transients in Drosophila. J Comp Physiol [A] 159:655-665.

Coburn CM, Bargmann CI (1996) A putative cyclic-nucleotide gated channel is required for sensory development and function in C. elegans. Neuron 17:695-706.

Coburn CM, Mori I, Yoshima Y, Bargmann CI (1998) A cyclic nucleotide-gated channel inhibits sensory axon outgrowth in larval and adult Caenorhabditis elegans: a distinct pathway for maintenance of sensory axon structure. Development 125:249-258.

Cramer KS, Sur M (1998) Nitric oxide as a signaling molecule in visual system development. Prog Brain Res 118:101-114.

Cramer KS, Sur M (1999) The neuronal form of nitric oxide synthase is required for pattern formation by retinal afferents in the ferret lateral geniculate nucleus. Dev Brain Res 116:79-86.

Cramer KS, Angelucci A, Hahm JO, Bogdanov MB, Sur M (1996) A role for nitric oxide in the development of the ferret retinogeniculate projection. J Neurosci 16:7995-8004.

Davenport RW, Kater SB (1992) Local increases in intracellular calcium elicit local filopodial responses in Helisoma neuronal growth cones. Neuron 9:405-416.

Elphick MR, Williams L, O'Shea M (1996) New features of the locust optic lobe: evidence of a role for nitric oxide in insect vision. J Exp Biol 199:2395-2407.

Ernst AF, Wu. HH, El-Fakahany EE, McLoon SC (1999) NMDA receptor-mediated refinement of a transient retinotectal projection requires nitric oxide. J Neurosci 19:229-235.

Farber DB, Brown DM, Lilley RN (1979) Cyclic nucleotide dependent protein kinase and the phosphorylation of endogenous proteins of retinal rod outer segments. Biochemistry 18:370-378.

Garrity PA, Rao Y, Salecker I, McGlade J, Pawson T, Zipursky SL (1996) Drosophila photoreceptor axon guidance and targeting requires the Dreadlocks SH2/SH3 adapter protein. Cell 85:639-650.

Gibbs SM (2001) Regulation of Drosophila visual system development by nitric oxide, cyclic GMP. Am Zool 41:268-281.

Gibbs SM, Truman JW (1998) Nitric oxide and cyclic GMP regulate retinal patterning in the optic lobe of Drosophila. Neuron 20:83-93.

Gibson NJ, Nighorn A (2000) Expression of nitric oxide synthase and soluble guanylate cyclase in the developing olfactory system of Manduca sexta. J Comp Neurol 422:191-205.

Gomez TM, Spitzer NC (1999) In vivo regulation of axon extension and pathfinding by growth-cone calcium transients. Nature 397:350-355.

Grigliatti T (1986) Mutagenesis. In: Drosophila: a practical approach (Roberts, DB, ed), pp 39-58. Oxford: IRL.

Harteneck C, Koesling D, Soling A, Schultz G, Bohme E (1990) Expression of soluble guanylate cyclase catalytic activity requires two enzyme subunits. FEBS Lett 272:221-223.

Hartzell HC, Fischmeister R (1986) Opposite effects of cyclic GMP and cyclic AMP on Ca2 + current in single heart cells. Nature 323:273-275.

Heisenberg M (1972) Comparative behavioral studies on two visual mutants of Drosophila. J Comp Physiol 80:119-136.

Hiesinger PR, Reiter C, Schau H, Fischbach KF (1999) Neuropil pattern formation and regulation of cell adhesion molecules in Drosophila optic lobe development depend on synaptobrevin. J Neurosci 19:7548-7556.

Hing H, Xiao J, Harden N, Lim L, Zipursky SL (1999) Pak functions downstream of Dock to regulate photoreceptor axon guidance in Drosophila. Cell 97:853-863.

Huang Z, Kunes S (1996) Hedgehog, transmitted along retinal axons, triggers neurogenesis in the developing visual centers of the Drosophila brain. Cell 86:411-422.

Huang Z, Shilo B-Z, Kunes S (1998) A retinal axon fascicle uses Spitz, and EGF receptor ligand, to construct a synaptic cartridge in the brain of Drosophila. Cell 95:693-703.

Inoue H, Yoshioka T, Hotta Y (1985) A genetic study of inositol trisphosphate involvement in phototransduction using Drosophila mutants. Biochem Biophys Res Commun 132:513-519.

Johnson EC, Robinson PR, Lisman JE (1986) Cyclic GMP is involved in the excitation of invertebrate photoreceptors. Nature 324:468-470.

Kafitz KW, Leinders-Zufall T, Zufall F, Greer CA (2000) Cyclic GMP evoked calcium transients in olfactory receptor cell growth cones. NeuroReport 111:677-681.

Karess RE, Rubin GM (1984) Analysis of P transposable elements functions in Drosophila. Cell 38:135-146.

Leamy CA, Ho-Pao CL, Sur M (2001) Disruption of retinogeniculate 
pattern formation by inhibition of soluble guanylate cyclase. J Neurosci 21:3871-3880.

Lee C-H, Herman T, Clandinin TR, Lee R, Zipursky SL (2001) $\mathrm{N}$-cadherin regulates target specificity in the Drosophila visual system. Neuron 30:437-450.

Liu W, Yoon J, Burg M, Chen L, Pak W (1995) Molecular characterization of two Drosophila guanylate cyclases expressed in the nervous system. J Biol Chem 270:12418-12427.

Meinertzhagen IA, Hanson TE (1993) The development of the optic lobe. In: The development of Drosophila melanogaster (Bate M, Arias AM, eds), pp 1363-1491. Plainview, NY: Cold Spring Harbor Laboratory.

Melzig J, Burg M, Gruhn M, Pak WL, Buchner E (1998) Selective histamine uptake rescues photo- and mechanoreceptor function of histidine decarboxylase-deficient Drosophila mutants. J Neurosci 18:7160-7166.

Miki N, Kawabe Y, Kuriyama K (1977) Activation of cerebral guanylate cyclase by nitric oxide. Biochem Biophys Res Commun 75:851-856.

Mize RR, Lo F (2000) Nitric oxide, impulse activity, and neurotrophins in visual system development. Brain Res 886:15-32.

Nawy S, Jahr CE (1990) Suppression by glutamate of cGMP-activated conductance in retinal bipolar cells. Nature 346:269-271.

Newsome TP, Schmidt S, Dietzl G, Keleman K, Asling B, Debant A, Dickson BJ (2000) Trio combines with Dock to regulate Pak activity during photoreceptor axon pathfinding in Drosophila. Cell 101:283-294

Nighorn A, Gibson NJ, Rivers DM, Hildebrand JG, Morton DB (1998) The nitric oxide-cGMP pathway may mediate communication between sensory afferents and projections neurons in the antennal lobe of Manduca sexta. J Neurosci 18:7244-7255

Ott SR, Jones IW, Burrows M, Elphick MR (2000) Sensory afferents and motor neurons as targets for nitric oxide in the locust. J Comp Neurol 422:521-532

Paupardin-Tritsch D, Hammond C, Gerschenfeld HM, Nairn AC, Greengard $\mathrm{P}$ (1986) cGMP-dependent protein kinase enhances $\mathrm{Ca} 2+$ current and potentiates the serotonin-induced $\mathrm{Ca} 2+$ current increase in snail neurons. Nature 323:812-814.

Polleux F, Morrow M, Ghosh A (2000) Semaphorin 3A is a chemoattractant for cortical apical dendrites. Nature 404:567-573.

Rao Y, Zipursky SL (1998) Domain requirements for the Dock adapter protein in growth-cone signaling. Proc Natl Acad Sci USA 95:2077-2082.

Rehder V, Kater SB (1992) Regulation of neuronal growth cone filipodia by intracellular calcium. J Neurosci 12:3175-3186.

Rockwell RF, Seiger MB (1973) Phototaxis in Drosophila: a critical evaluation. Am Sci 61:339-345.

Sauzeau V, Le Jeune H, Cario-Toumaniantz C, Smolenski A, Lohmann SM, Bertoglio J, Chardin P, Pacaud P, Loirand G (2000) Cyclic GMPdependent protein kinase signaling pathway inhibits RhoA-induced $\mathrm{Ca}^{2+}$ sensitization of contraction in vascular smooth muscle. J Biol Chem 275:21722-21729.

Sawada N, Itoh H, Yamashita J, Doi K, Inoue M, Masatsugu K, Fukunaga Y, Sakaguchi S, Stone M Yamahara KK, Yuguri T, Nakao K (2001) cGMP-dependent protein kinase phosphorylates and inactivates RhoA. Biochem Biophys Res Commun 280:789-805.

Schachtner J, Klaassen L, Truman JW (1998) Metamorphic control of cyclic guanosine monophosphate expression in the nervous system of the tobacco hornworm, Manduca sexta. J Comp Neurol 396:238-252.
Schmachtenberg O, Bicker G (1999) Nitric oxide and cyclic GMP modulate photoreceptor cell responses in the visual system of the locust. J Exp Biol 202:13-20.

Schneider T, Reiter C, Eule E, Bader B, Lichte B, Nie Z, Schimansky T, Ramos RG, Fischbach KF (1995) Restricted expression of the irreCrst protein is required for normal axonal projections of columnar visual neurons. Neuron 15:259-271.

Shah S, Hyde DR (1995) Two Drosophila genes that encode the $\alpha$ and $\beta$ subunits of the brain soluble guanylyl cyclase. J Biol Chem 270:15368-15376.

Shieh B-H, Zhu M-Y, Lee JK, Kelly IM, Bahiraei F (1997) Association of INAD with NORPA is essential for controlled activation and deactivation of Drosophila phototransduction in vivo. Proc Natl Acad Sci USA 94:12682-12687.

Song HJ, Ming GL, He Z, Lehmann M, McKerracher L, Tessier-Lavigne M, Poo M-M (1998) Conversion of neuronal growth cone responses from repulsion to attraction by cyclic nucleotides. Science 281:1515-1518.

Surks HK, Mochizuki N, Kasai Y, Georgescu SP, Tang KM, Ito M, Lincoln TM, Mendelsohn ME (1999) Regulation of myosin phosphatase by a specific interaction with cGMP-dependent protein kinase I alpha. Science 286:1583-1587.

Thummel C, Pirrotta C (1992) Technical notes: new pCaSpeR P element vectors. Drosoph Inf Serv 71:150.

Truman JW, De Vente J, Ball EE (1996) Nitric oxide-sensitive guanylate cyclase activity is associated with the maturational phase of neuronal development in insects. Development 122:3949-3958.

Van Wagenen S, Rehder V (1999) Regulation of neuronal growth cone filipodia by nitric oxide. J Neurobiol 39:168-185.

Van Wagenen S, Rehder V (2000) Regulation of neuronal growth cone filipodia by nitric oxide depends on soluble guanylate cyclase. J Neurobiol 46:206-219.

Wildemann B, Bicker G (1999) Developmental expression of nitric oxide/cyclic GMP synthesizing cells in the nervous system of Drosophila melanogaster. J Neurobiol 38:1-15.

Wolff T, Martin KA, Rubin GL, Zipursky SL (1997) The development of the Drosophila visual system. In: Molecular and cellular approaches to neural development (Cowan WM, Jessell TM, Zipursky SL, eds), pp 474-508. New York: Oxford UP.

Wu HH, Williams CV, McLoon SC (1994) Involvement of nitric oxide in the elimination of a transient retinotectal projection in development Science 265:1593-1596.

Wu HH, Cork RJ, Huang PL, Shuman DL, Mize RR (2000) Refinement of the ipsilateral retinocollicular projection is disrupted in double endothelial and neuronal nitric oxide synthase gene knockout mice. Brain Res Dev Brain Res 120:105-111.

Yoshikawa S, Miyamoto I, Aruga J, Furuichi T, Okano H, Mokoshiba K (1993) Isolation of a Drosophila gene encoding a head-specific guanylyl cyclase. J Neurochem 60:1570-1573.

Zayas RM, Qazi S, Morton DB, Trimmer BA (2000) Neurons involved in nitric-oxide mediated cGMP signaling in the tobacco hornworm, Manduca sexta, J Comp Neurol 419:422-438.

Zipursky SL, Venkatesh TR, Teplow DB, Benzer S (1984) Neuronal development in the Drosophila retina: monoclonal antibodies as molecular probes. Cell 3:615-616.

Zuker CS (1996) The biology of vision in Drosophila. Proc Natl Acad Sci USA 93:571-576. 